\title{
A Distributed Multi-Competitive Clustering Method for Wireless Sensor Networks using 5G LTE
}

\author{
G. Suprita Reddy, V. Poornima
}

\begin{abstract}
Fifth era remote, or $5 G$, is the most recent emphasis of cell innovation, designed to extraordinarily build the speed and responsiveness of remote systems. With 5G, information transmitted over remote broadband associations could go at rates as high as 20 Gbps by a few appraisals surpassing wire line organize speeds and in addition offer inactivity of $1 \mathrm{~ms}$ or lower for utilizes that require ongoing criticism. 5G will likewise empower a sharp increment in the measure of information transmitted over remote frameworks because of more accessible transfer speed and propelled reception apparatus technology. Wireless sensor systems (WSNs) require basic and successful ways to deal with lessen vitality utilization in view of restricted vitality. Grouping hubs is a compelling way to deal with make WSNs vitality productive. In this paper we propose a dispersed multi-aggressive bunching approach (DMCC) for WSNs. To start with, the hubs with high leftover vitality are chosen to go about as group head hopefuls (CHCs). Second, bunch heads (CHs) are chosen from the CHCs in light of a crossover of rivalry. In the event that the separations to the chose $\mathrm{CHs}$ are reasonable, a CHC with more neighbour hubs and littler normal separation to its neighbour hubs will probably turn into a $\mathrm{CH}$. In the event that the quantity of $\mathrm{CH}$ shose from the $\mathrm{CHCs}$ is insufficient, more CHs are chosen from non-CHCs constantly as indicated by lingering vitality until the CHs number is reasonable. DMCC makes the CHs number stable and disperse the CHs equally. Recreation tests were performed on to contrast DMCC and some related bunching approaches. The exploratory outcomes recommend that DMCC adjusts the heap among various groups and lessens the vitality utilization, which enhances the system lifetime.
\end{abstract}

\section{INTRODUCTION}

Not with standing enhancements in speed, limit and idleness, 5G offers arrange administration highlights, among them organize cutting, which enables versatile administrators to make various virtual systems inside a solitary physical 5G arrange. This capacity will empower remote system associations with help particular uses or business cases and could be sold on an as-a-benefit premise. A self-driving auto, for instance, would require a system cut that offers to a great degree quick, low-idleness associations so a vehicle could explore continuously. A home machine, be

Revised Manuscript Received on 14 August, 2019.

Mrs.G.Suprita Reddy, Asst. Professor, Dept. of ECE, Malla Reddy Engineering College for Women (Autonomous), Secunderabad-TS suprita542@gmail.com

Mrs V.Poornima, Asst. Professor, Dept. of ECE, Malla Reddy Engineering College for Women (Autonomous), Secunderabad-TS poornima40427@gmail.com ${ }^{2}$ that as it may, could be associated through a lower-control, slower association since superior isn't essential. The web of things (IoT) could utilize secure, information just connections.5G systems and administrations will be conveyed in stages finished the following quite a while to oblige the expanding dependence on portable and web empowered gadgets. Generally, 5G is relied upon to create an assortment of new applications, uses and business cases as the innovation is taken off.

Remote systems are made out of cell locales isolated into parts that send information through radio waves. Fourth-age (4G) Long-Term Evolution (LTE) remote innovation gives the establishment to $5 \mathrm{G}$. Not at all like $4 \mathrm{G}$, which requires vast, high-control cell towers to emanate motions over longer separations, $5 \mathrm{G}$ remote signs will be transmitted by means of substantial quantities of little cell stations situated in places like light shafts or building rooftops. The utilization of numerous little cells is vital on the grounds that the milli meter wave range - the band of range between $30 \mathrm{GHz}$ and $300 \mathrm{GHz}$ that $5 \mathrm{G}$ depends on to produce high speeds can just go over short separations and is liable to obstruction from climate and physical hindrances, similar to structures. Past ages of remote innovation have utilized lower-recurrence groups of range. To balance milli meter wave challenges identifying with separation and impedance, the remote business is likewise considering the utilization of lower-recurrence range for $5 \mathrm{G}$ organizes so arrange administrators could utilize range they effectively possess to work out their new systems.

\section{RELATED WORKS}

Scientists have considered grouping structures of WSNs for a long time. Filter is a normal bunching convention. This convention chooses a few hubs as CHs. A hub which isn't a $\mathrm{CH}$ picks a $\mathrm{CH}$ to join the group and sends information to the $\mathrm{CH}$. CHs get and total information, at that point send information to BS. Filter adjusts the hubs vitality utilization, and delays the system lifetime. Drain has the benefits of straightforward and compelling, however have generous issues moreover. One issue is that the $\mathrm{CHs}$ are chosen arbitrarily which makes the grouping inconsistent. Because of the arbitrariness display in bunching, the quantity of $\mathrm{CHs}$ created differs profoundly from the ideal number. The circulation of $\mathrm{CHs}$ is uneven, and the distinction of the hubs number among bunches might

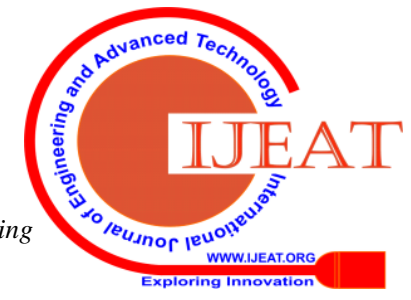




\section{A Distributed Multi-Competitive Clustering Method for Wireless Sensor Networks using 5G LTE}

be substantial. Clearly, vitality utilization is distinctive between a $\mathrm{CH}$ with bunches of hubs in its group and a $\mathrm{CH}$ with just couple of hubs in its group. Distinctive vitality utilization of CHs isn't helpful for the expansion of the system lifetime. Drain MAC tries to control the arbitrariness introduce in LEACH. This approach makes the $\mathrm{CH}$ number stable and ascertains the ideal $\mathrm{CH}$ s number. During the time spent $\mathrm{CH}$ s choice, the quantity of chose $\mathrm{CHs}$ is recorded, and the choice procedure is end until the point that the quantity of chose $\mathrm{CHs}$ accomplishes the ideal number. LEACHMAC enhances the general system lifetime. While figuring the ideal $\mathrm{CHs}$ number, LEACH-MAC expect that $\mathrm{CHs}$ are conveyed equally, yet the calculation does not consider how to disseminate $\mathrm{CHs}$ uniformly, so genuine $\mathrm{CHs}$ circulation is clearly not quite the same as the perfect, and the appropriate $\mathrm{CHs}$ number is unique in relation to the ascertained number. To draw out the system lifetime, $\mathrm{CHs}$ can be chosen by the remaining vitality of hubs . Ref takes motivation from organic frameworks to build up a honey bee propelled convention that is vitality mindful, versatile and productive. EDCS decides the likelihood of a hub to be a $\mathrm{CH}$ through normal system leftover vitality estimation as per the normal system vitality utilization conjecture in perfect state and reference estimation of chronicled vitality utilization at the same time. Here proposes a CHs determination component in light of the gauge of the hub remaining vitality and a resting planning model. Notice chooses beginning competitors in light of leftover vitality. A hub with higher remaining vitality will probably turn into an underlying hopeful. At that point the quantity of neighbor hubs or closeness to the neighbour hubs is utilized to choose $\mathrm{CHs}$ in the underlying competitors. Notice confines the base separation between $\mathrm{CHs}$, yet the separation between $\mathrm{CHs}$ might be too far. When it happens, a hub may finish HEED execution without choosing a $\mathrm{CH}$ and it must turn into a $\mathrm{CH}$, so the quantity of $\mathrm{CHs}$ shifts in various round WRECS takes the standardized lingering vitality as $\mathrm{CHs}$ choice parameters. The quantity of the hubs in a bunch is spoken to by m. WRECS trusts that the vitality utilization of a $\mathrm{CH}$ is relative to $\mathrm{m}$.

Once a hub has been a $\mathrm{CH}$, it lost the opportunity to go about as a $\mathrm{CH}$ again for $\mathrm{km}$ rounds. Here, $\mathrm{k}$ is a steady. In the hubs that have the opportunity to be $\mathrm{CHs}, \mathrm{CHs}$ are chosen by the remaining vitality. Since $\mathrm{CH}$ vitality utilization isn't altogether controlled by m, WRECS can't totally adjust the vitality utilization among CHs. WRECS put off the begin time of hub demise to a specific degree, however the calculation can't lessen vitality utilization of the entire system. DUCF shapes unequal bunches to adjust the vitality utilization among the $\mathrm{CHs}$. The calculation gauges the most extreme number of hubs in a group. At the point when a $\mathrm{CH}$ discovers its own particular group part to accomplish the most extreme number, it never again acknowledges new hubs to join the bunch. DUCF adjusts the quantities of hubs among groups, so the vitality utilizations of $\mathrm{CHs}$ are adjusted, and the system lifetime is delayed. Be that as it may, DUCF experiences a few issues.

The bunching procedure is unpredictable. A few hubs may encounter the procedures for a few times: select a $\mathrm{CH}$ join the group disappointment reselect another $\mathrm{CH}$. Now and again a hub needs to choose a removed $\mathrm{CH}$, or straightforwardly speak with BS, which will build the vitality utilization. When all is said in done, these techniques all remain imperfect. We propose DMCC in this paper. Alongside lingering vitality and number of neighbour hubs, ideal $\mathrm{CHs}$ number, ideal separation amongst $\mathrm{CHs}$ and normal separation between the hubs and their $\mathrm{CH}$ are likewise used to choose $\mathrm{CHs}$.

\section{NETWORKS AND RADIO MODEL}

In this paper, we accept a WSN that covers a square region. The side length of the square is spoken to by $M$. Every hub has the accompanying properties:

(a) All hubs are equally circulated and settled.

(b) BS is appropriated at the focal point of the zone, and it is settled.

(c) Nodes send information to CHs by single-jump, and CHs send information to BS by single-bounce.

(d) A hub knows about itself lingering vitality yet unconscious of position.

(e) All hubs can total, send and get information, and the transmission power can be versatile. A hub can recognize the quality of get flag, so a recipient can gauge the separation to the sender by contrasting it and the quality of sends the flag.

\section{PROPOSED APPROACH}

Altered DMCC is a volte LEACH-like grouping approach which is separated into bunches development and information transmission. ALL hubs are divided into a few bunches and each group has one $\mathrm{CH}$. The hubs assemble and transmit information to their $\mathrm{CHs}$. $\mathrm{CHs}$ get and total information at that point sends information to BS. The determination of $\mathrm{CHs}$ is completed in three stages. To start with we select a few hubs with high leftover vitality to be CHCs. Second $\mathrm{CHs}$ are chosen by rivalry among $\mathrm{CHCs}$ as indicated by the quantity of the neighbour hubs, the normal separation to the neighbour hubs and the separations between the $\mathrm{CHC}$ and the $\mathrm{CHs}$ that has been chosen as of now; if the quantity of CHs chose at the second step is not as much as the ideal number, third steps must be done to choose more $\mathrm{CHs}$ as a supplement.

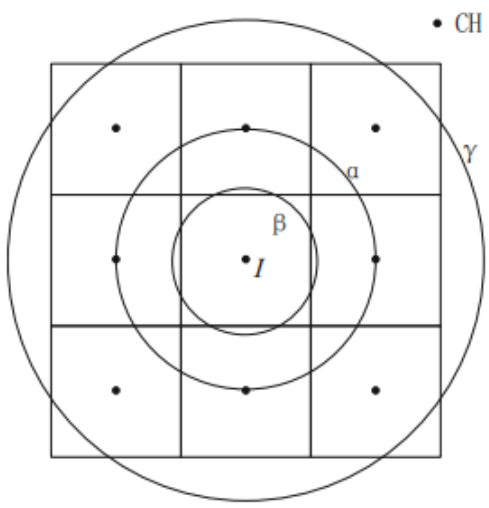

Fig: 1 optimal cluster setup

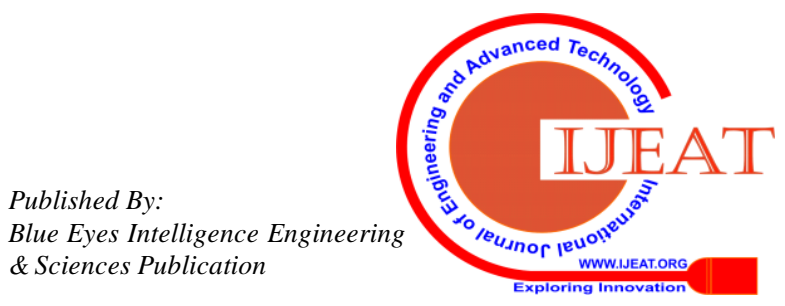




\subsection{The Main Steps of DMCC}

The particular advances are as per the following:

Stage 1: At the first run through, every hub communicates its vitality and ID with given flag quality. A hub gets signals from different hubs and identifies the quality of getting signs to assess the separations between the senders and it done. At that point, a hub figures $\mathrm{Aj}$ and $\mathrm{Bj}$.

Stage 2: BS gets the signs and influences a remaining vitality to succession to get Embed, at that point communicates Embed to all hubs and begin $\mathrm{t} 1$ ? $\mathrm{t} 2$ timing.

Stage 3: each hubs gets the message from BS, chooses whether to wind up a CHC. Each $\mathrm{CHC}$ computes t $\mathrm{j}$ and begin timing. Different hubs ascertain ti ? $\mathrm{t} 1$ and begin timing.

Stage 4: If a hub gets a group frame message amid pausing, it evaluates the separation between the sender and itself. On the chance that the separation is the hub loses the opportunity to be a $\mathrm{CH}$ for the current round. When holding up time is finished, if a hub still has the opportunity to be a $\mathrm{CH}$ and the $\mathrm{CHs}$ choice isn't end, it turns into a $\mathrm{CH}$ for the current round and communicate a bunch shape message with its ID to different hubs.

Stage 5: If BS has gotten k group frame messages or $\mathrm{t} 1$ ? $\mathrm{t} 2$ is finished, $\mathrm{BS}$ communicates a $\mathrm{CHs}$-determination end message to end the $\mathrm{CHs}$ choice.

Stage 6: A hub gets the group frame messages. On the off chance that it's anything but a $\mathrm{CH}$ after the finish of $\mathrm{CHs}$ determination, it picks the nearest $\mathrm{CH}$ to send a participate in-message with the CHs ID and its ID. The CHs get the participate in-messages and acknowledge the solicitations. At that point the $\mathrm{CHs}$ present the acknowledge message on the hubs. Thus, a bunch is shaped.

Stage 7: Nodes accumulate information and transmit the information with their lingering vitality data to their CHs. $\mathrm{CHs}$ get and total the information frame the hubs, at that point, transmit the information with the leftover vitality data to BS. BS gets the information. At the point when a round is end, rehash from stage 2 .

\section{RESULTS AND ANALYSIS}

We re-enacted DMCC in the matlab condition. The recreation comes about are contrasted and LEACH, LEACH-MAC and DUCF in various net-work situations. Situation 1:200 hubs are equally scattered in a square district of size $150 \mathrm{~m} 9150 \mathrm{~m}$ and BS is situated at the inside. Situation 2: 200 hubs are uniformly scattered in a square locale of size $150 \mathrm{~m} 9150 \mathrm{~m}$ and BS is outside to the district. Situation 3: 200 hubs are haphazardly scattered in a square district of size $150 \mathrm{~m} 9150 \mathrm{~m}$ and BS is situated at the middle. Situation 4: 300 hubs are equitably scattered in a square locale of size $200 \mathrm{~m} 9200 \mathrm{~m}$ and BS is situated at the middle.

In this section, we compare our algorithm with five baseline algorithms in terms of six clustering indexes, i.e., NMI, AVI, F-scores, Recall, Purity, Classification Rate (CR), AMI, on two data sets (Mfeat, Image Segmentation(IS)). When all the clustering indexes are higher, the better clustering result performs. The five baseline algorithms are summarized below:

\subsection{Comparison with different calculations}

In this area, we contrast our calculation and five pattern calculations regarding six grouping files, i.e., NMI, AVI, F-scores, Recall, Purity, Classification Rate (CR), AMI, on two informational collections ( $\mathrm{M}$ feat, Image Segmentation(IS)). At the point when all the bunching records are higher, the better grouping outcome performs. The five gauge calculations are condensed beneath:

- EW-k-implies: EW-k-implies is a weighted $\mathrm{k}$ implies bunching which can consequently take in the weights of the highlights as per their significance.

- Feat Concate: Feature Concatenation calculation executes the ghostly grouping in the wake of investigating the highlights of each view and linking the highlights.

- WVCMM: WVCMM is a weighted multi-see bunching called weighted multi-see CMM (convex blend models) which accept that the information in each view depend on the Gaussian conveyance.

- Co-prepared Spectral grouping: Co-prepared Spectral bunching is a calculation under co-preparing system going for achieving an understanding over the perspectives. The calculation utilizes the names in a single view and takes in the marks in different perspectives.

- TW-k-implies: TW-k-implies (Two-Level variable weighting k-implies calculation) can be viewed as an expansion to the $\mathrm{k}$-implies by including the view weights and the element weights in computing

- WVCMM: WVCMM is a weighted multi-view clustering called weighted multi-view CMM (convex mixture models) which assume that the data in each view are based on the Gaussian distribution.

- Co-trained Spectral clustering: Co-trained Spectral clustering is an algorithm under co-training framework aiming at reaching an agreement across the views. The algorithm uses the labels in one view and learns the labels in other views.

- TW-k-means: TW-k-means (Two-Level variable weighting k-means algorithm) can be seen as an extension to the k-means by adding the view weights and the
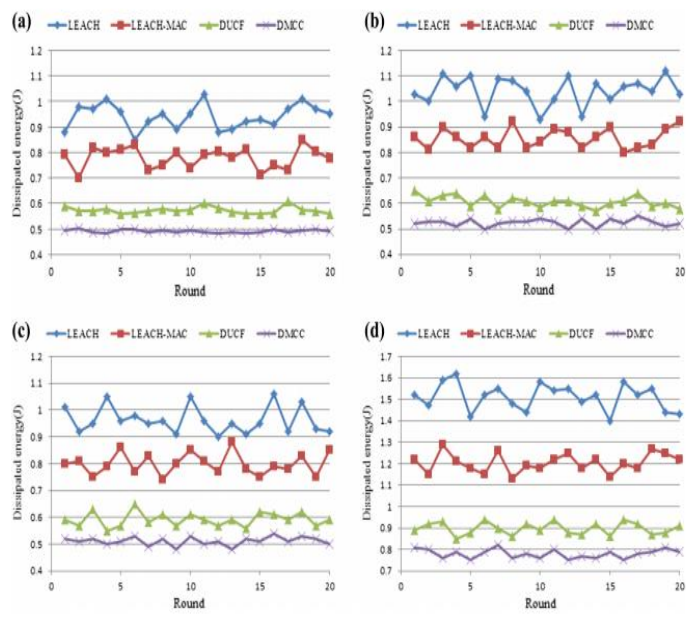

Fig. 3: Dissipated energy by all notes for 20 rounds. a Scenario 1, b Scenario 2, c Scenario 3, d Scenario 4

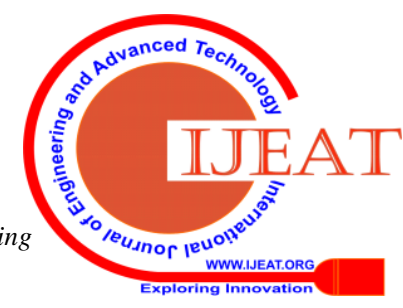


Drain MAC diminishes the entire system vitality utilization to draw out the system lifetime, yet the haphazardness of $\mathrm{CHs}$ determination may prompt improper bunching, particularly when the hubs are arbitrarily appropriated in situation 3, so the lifetime is still short.

\section{CONCLUSIONS}

In this paper, we have proposed a new algorithm for multi-view clustering which exploits the weighted multi view algorithm strategy and on-line learning technique. Additionally, we add the sparsely norm and the competitive learning to the algorithm which make the algorithm converge to minima faster and perform a more accurate result. We use the alterative optimization method to optimize the objective function.

Experiments on two real-world data sets indicate the improvement and effectiveness of our method. In the future work, we will consider the way automatically determining the parameters of the algorithm as well as automatically determining the initial centre. Moreover, we will research on combining variable weighting method with other clustering techniques for reaching a better clustering result.

\section{REFERENCES}

1. X. Zhao, N. Evans, and J.-L. Dugelay, "Multi-view k-means clustering on big data," Pattern Recognition Letters, vol. 41, pp. 73-82, 2014.

2. G. Tzortzis and A. Likas, "Kernel-based weighted multi-view clustering," in ICDM, 2012, pp. 675-684.

3. X. Cai, F. Nie, and H. Huang, "Multi-view k-means clustering on big data," in IJCAI, 2013, pp. 2598-2604.

4. X. Chen, X. Xu, J.Z, Huang, and Y. Ye, "Tw-k-means: Automated two-level variable weighting clustering algorithm for multiview data," IEEE Trans. KDE, vol. 25, pp. 932-944, 2013.

5. C.-D. Wang and J.-H. Lai, "Energy based competitive learning," Neurocomputing, vol. 74, pp. 2265-2275, 2011

6. L. $\mathrm{Xu}$, "Rbf nets, mixture experts, and bayesian ying-yang learning," Neurocomputing, vol. 19, pp. 223-257, 1998.

7. R. M. Gray, "Vector quantization," IEEE ASSP Magazine, vol. 1, pp. 4-29, 1984.

8. L. tak Law and Y. ming Cheung, "Color image segmentation using rival penalized controlled competitive learning," in IJCNN, vol. 4, 2003, pp. $108-112$.

9. A. Banerjee and J. Ghosh, "Frequency sensitive competitive learning for balanced clustering on high-dimensional hyperspheres," IEEE Trans. NN, vol. 15, pp. 702-719, 2004.

10. L. Xu, A. Krzyzak, and E. Oja, "Rival penalized competitive learning for clustering analysis, rbf net, and curve detection," IEEE Trans. NN, vol. 4, pp. 636-649, 1993.

11. . N. Pantazis, S. Nikolidakis and D. Vergados, Energy efficient routing protocols in wireless sensor networks: A survey, IEEE Communications Surveys \& Tutorials, Vol. 15, No. 2, pp. 551-591, 2013

12. H. Sivasankari, R. Leelavathi and M. Vallabh, CEAR: Cluster based Energy Aware Routing algorithm to maximize lifetime of Wireless Sensor Networks, SpringerNew York, 2013. pp. 31-37.

13. M. Saleem, I. Ullah and M. Farooq, Bee Sensor: An energy-efficient and scalable routing protocol for wireless sensor networks, Information Sciences, Vol. 200, No. 2, pp. 38-56, 2012.

14. Y. Yongjian, J. Bing and W. Jie, An Improved Algorithm for LEACH Protocol in Wireless Sensor Network, Journal of Beijing University of Posts and Telecommunications, Vol. 36, No. 1, pp. 105-109, 2013

15. O. Younis and S. Fahmy, Heed: A hybrid, energy-efficient, distributed clustering approach for ad hoc sensor networks, IEEE Transactions on Mobile Computing, Vol. 3, No. 4, pp. 366-379, 2004. 